Agradecemos aos pareceristas que contribuíram em 2021 para assegurar o padrão de qualidade de Cadernos de Pesquisa.

\section{Adelina Novaes}

Fundação Carlos Chagas - FCC,

São Paulo (SP), Brasil

\section{Adriano Souza Senkevics}

Instituto Nacional de Estudos e Pesquisas Educacionais

Anisio Teixeira - Inep,

Brasilia (DF), Brasil

Agda Malheiro Ferraz de Carvalho

Pontifícia Universidade Católica de São Paulo - PUC-SP,

São Paulo (SP), Brasil

Ailton Barcelos da Costa

Universidade Federal de São Carlos - UFSCar,

São Carlos (SP), Brasil

Alice Miriam Happ Botler

Universidade Federal de Pernambuco - UFPE,

Recife (PE), Brasil

Alisson Machado

Universidade Federal de Santa Maria - UFSM,

Florianópolis (SC), Brasil

Almerindo Janela Afonso

Universidade do Minho - UMinho,

Braga, Portugal

Amélia Cristina Abreu Artes

Fundação Carlos Chagas - FCC,

São Paulo (SP), Brasil

Ana Beatriz Gomes Pimenta de Carvalho Universidade Federal de Pernambuco - UFPE, Recife (PE), Brasil

\section{Ana Castro Zubizarreta}

Universidad de Cantabria - Unican,

Cantabria, Espanha

Ana Cláudia ChavesTeixeira

Universidade de Campinas - Unicamp,

Campinas (SP), Brasil
Ana Maria Mauad

Universidade Federal Fluminense - UFF,

Niterói (RJ), Brasil

Analía Otero

Faculdade Latino-Americana de Ciências Sociais - Flacso,

Buenos Aires, Argentina

Andreza Barbosa

Centro Universitário Salesiano de São Paulo - Unisal,

Americana (SP), Brasil

Ângela de Fátima Saraiva Freitas

Instituto Nacional de Câncer - Inca,

Rio de Janeiro (RJ), Brasil

Ângela Maria Scalabrin Coutinho

Universidade Federal do Paraná- UFPR,

Curitiba (PR), Brasil

Angela Maria Martins

Fundação Carlos Chagas - FCC,

São Paulo (SP), Brasil

Ângelo Ricardo de Souza

Universidade Federal do Paraná - UFPR,

Curitiba (PR), Brasil

Anna Carolina Venturini

Universidade do Estado do Rio de Janeiro - UERJ,

Rio de Janeiro (RJ), Brasil

Antonio Alvaro Soares Zuin

Universidade Federal de São Carlos - UFSCar,

São Carlos (SP), Brasil

Arnaldo Lôpo Mont'Alvão Neto

Universidade do Estado do Rio de Janeiro - UERJ,

Rio de Janeiro (RJ), Brasil

Barbara Cobo Soares

Instituto Brasileiro de Geografia e Estatística - IBGE, Rio de Janeiro (RJ), Brasil 
Beatriz Giugliani

Universidade Federal do Recôncavo da Babia - UFRB,

Cruz das Almas (BA), Brasil

Bernardete A. Gatti

Conselho Estadual de Educação de São Paulo - CEE-SP,

São Paulo (SP), Brasil

Bianca Margarita Damin Tavolari

Insper, São Paulo (SP), Brasil

Bruno Bontempi Jr.

Universidade de São Paulo - USP,

São Paulo (SP), Brasil

Carlos Roberto Jamil Cury

Pontifícia Universidade Católica de Minas Gerais - PUC-MG,

Belo Horizonte (MG), Brasil

Carolina Aparicio Molina

Universidad Católica de la Santísima Concepción - UCSC,

Concepcion, BioBio, Chile

Cassia Geciauskas Sofiato

Universidade de São Paulo - USP,

São Paulo (SP), Brasil

Célia Auxiliadora dos Santos Marra

Centro de Apoio ao Desenvolvimento Humano Integral Ltda. - Ceadi,

Belo Horizonte (MG), Brasil

Claudia Dias Prioste

Universidade Estadual Paulista "Júlio de Mesquita Filho" - Unesp,

Araraquara (SP), Brasil

Claudia Rosa Riolfi

Universidade de São Paulo - USP,

São Paulo (SP), Brasil

Claudio Pinto Nunes

Universidade Estadual do Sudoeste da Babia - UESB,

Vitória da Conquista (BA), Brasil

Cleomar Locatelli

Universidade Federal do Tocantins - UFT,

Palmas (TO), Brasil

Cristián Cox Donoso

Universidad Diego Portales - UDP,

Santiago, Chile

Cristiane da Silva Cabral

Universidade de São Paulo - USP,

São Paulo (SP), Brasil
Cristina Rodrigues da Silva

Universidade Federal de São Carlos - UFSCar,

São Carlos (SP), Brasil

Cynthia María Torres Stockl

Universidad Nacional de Tucumán - UNT,

Tucumán, Argentina

Dalila Andrade Oliveira

Universidade Federal de Minas Gerais - UFMG,

Belo Horizonte (MG), Brasil

Débora Cristina de Araújo

Universidade Federal do Espirito Santo - UFES,

Vitória (ES), Brasil

Diana Gonçalves Vidal

Universidade de São Paulo - USP,

São Paulo (SP), Brasil

Diego Miguel-Revilla

Universidad de Valladolid - UVa,

Valladolid, España

Dieison Marconi

Universidade Federal do Rio Grande do Sul-UFRGS,

Porto Alegre (RS), Brasil

Domingos Manuel Barros Fernandes

Instituto Universitário de Lisboa - ISCTE,

Lisboa, Portugal

Dyane Brito Reis Santos

Universidade Federal do Recôncavo da Babia - UFRB,

Cruz das Almas (BA), Brasil

Edivaldo Herculano Corrêa de Oliveira

Instituto Evandro Chagas - IEC,

Ananindeua (PA), Brasil

Elaine Conte

Universidade La Salle - Unilasalle,

Canoas (RS), Brasil

Elba Siqueira de Sá Barretto

Universidade de São Paulo - USP,

São Paulo (SP), Brasil

Eliane Rose Maio

Universidade Estadual de Maringá - UEM,

Maringá (PR), Brasil

Elisabeth Meloni Vieira

Universidade de São Paulo - USP,

São Paulo (SP), Brasil 
Elizabeth Balbachevsky

Universidade de São Paulo - USP,

São Paulo (SP), Brasil

Eloisa Maia Vidal

Universidade Estadual do Ceará - UECE,

Fortaleza (CE), Brasil

Eva Blay

Universidade de São Paulo - USP,

São Paulo (SP), Brasil

Fabiana Silva Fernandes

Fundação Carlos Chagas - FCC,

São Paulo (SP), Brasil

Fabiane Ferreira da Silva

Universidade Federal do Pampa - Unipampa,

Bagé (RS), Brasil

Fábio Domingues Waltenberg

Universidade Federal Fluminense - UFF,

Niterói (RJ), Brasil

Fernanda Nascimento da Silva

Universidade Federal de Santa Catarina - UFSC,

Florianópolis (SC), Brasil

Flavia Medeiros Sarti

Universidade Estadual Paulista "Júlio de Mesquita Filho" - Unesp,

Rio Claro (SP), Brasil

Flávia Milena Biroli Tokarski

Universidade de Brasilia - UnB,

Brasilia (DF), Brasil

Flavia Pereira Xavier

Universidade Federal de Minas Gerais - UFMG,

Belo Horizonte (MG), Brasil

Franciele Bete Petry

Universidade Federal de Santa Catarina - UFSC,

Florianópolis (SC), Brasil

Gabriela Abuhab Valente

Universidade de São Paulo - USP,

São Paulo (SP), Brasil

Gabriela de Brito Caruso

Universidade do Estado do Rio de Janeiro - UERJ,

Rio de Janeiro (RJ), Brasil

Geilsa Costa Santos Baptista

Universidade Estadual de Feira de Santana - UEFS,

Feira de Santana (BA), Brasil
Girlene Ribeiro de Jesus

Universidade de Brasilia - UnB,

Brasilia (DF), Brasil

Gisela Lobo B. P. Tartuce

Fundação Carlos Chagas - FCC,

São Paulo (SP), Brasil

Giselle Aparecida de Athayde Massi

Universidade Tuiuti do Paraná-UTP,

Curitiba (PR), Brasil

Gladys Beatriz Barreyro

Universidade de São Paulo - USP, São Paulo (SP), Brasil /

Boston College, Boston, Massachusetts, Estados Unidos

Glaucia da Silva Brito

Universidade Federal do Paraná - UFPR,

Curitiba (PR), Brasil

Gustavo Passos

Universidade Federal do Rio Grande do Sul - UFRGS,

Porto Alegre (RS), Brasil

Isabel Pauline Hildegard Georges

Institut de Recherche pour le Développement - IRD, Paris, Françal

Universidade Federal de São Carlos - UFSCar,

São Carlos (SP), Brasil

Iza Rodrigues da Luz

Universidade Federal de Minas Gerais - UFMG,

Belo Horizonte (MG), Brasil

Jader Jane Moreira Lopes

Universidade Federal de Juiz de Fora - UFJF,

Juiz de Fora $(M G)$, Brasil

Javier Núñez Moscoso

Université de Montréal - UdeM,

Québec, Canada

Jefferson Mainardes

Universidade Estadual de Ponta Grossa-UEPG,

Ponta Grossa (PR), Brasil

Joana Paulin Romanowski

Pontifícia Universidade Católica do Paraná - PUC-PR,

Curitiba (PR), Brasil

Jorge Lyra

Universidade Federal de Pernambuco - UFPE,

Recife (PE), Brasil

José Machado Pais

Universidade de Lisboa - ULisboa,

Lisboa, Portugal 
Juan Rafael Muñoz Muñoz

Universidad de Almería - UAL,

Almeria, Espanha

Julio Groppa Aquino

Universidade de São Paulo - USP,

São Paulo (SP), Brasil

Julliane Messias Cordeiro Sampaio

Centro Universitário de Brasilia - CEUB,

Brasilia (DF), Brasil

Jussara Cristina Barboza Tortella

Pontificia Universidade Católica de Campinas - PUC-Campinas,

Campinas (SP), Brasil

Jutta Cornelia Reuwsaat Justo

Universidade Luterana do Brasil - Ulbra,

Canoas (RS), Brasil

Kaizô Iwakami Beltrão

Fundação Getúlio Vargas - FGV,

Rio de Janeiro (RJ), Brasil

Katya Mitsuko Zuquim Braghini

Pontifícia Universidade Católica de São Paulo - PUC-SP,

São Paulo (SP), Brasil

Kelly Russo

Universidade do Estado do Rio de Janeiro - UERJ,

Rio de Janeiro (RJ), Brasil

Lenira Haddad

Universidade Federal de Alagoas - UFAL,

Maceió (AL), Brasil

Liane Maria Bertucci

Universidade Federal do Paraná - UFPR,

Curitiba (PR), Brasil

Licínio C. Lima

Universidade do Minho - UMinho,

Braga, Portugal

Lisandra Príncipe

Universidade Paulista - Unip,

São Paulo (SP), Brasil

Livia de Araújo Donnini Rodrigues

Universidade de São Paulo - USP,

São Paulo (SP), Brasil

Lívia Maria Fraga Vieira

Universidade Federal de Minas Gerais - UFMG,

Belo Horizonte (MG), Brasil
Loriane Trombini Frick

Universidade Federal do Paraná - UFPR,

Curitiba (PR), Brasil

Luana Costa Almeida

Universidade Federal de São Carlos - UFSCar,

São Carlos (SP), Brasil

Lucídio Bianchetti

Universidade do Estado de Santa Catarina - Udesc,

Florianópolis (SC), Brasil

Lucíola Licínio de Castro Paixão Santos

Universidade Federal de Minas Gerais - UFMG,

Belo Horizonte (MG), Brasil

Luiz Alexandre da Silva Rosado

Instituto Nacional de Educação de Surdos - INES,

Rio de Janeiro (RJ), Brasil

Luiz Augusto Campos

Universidade do Estado do Rio de Janeiro - UERJ,

Rio de Janeiro (RJ), Brasil

Marcelo Pagliosa Carvalho

Universidade Federal do Maranhão - UFMA,

São Luis (MA), Brasil

Marcus Vinícius da Cunha

Universidade de São Paulo - USP,

Ribeirão Preto (SP), Brasil

Maria Clara Di Pierro

Universidade de São Paulo - USP,

São Paulo (SP), Brasil

\section{Maria Cláudia Cardoso Ferreira \\ Universidade da Integração Internacional da \\ Lusofonia Afro-Brasileira (Unilab), \\ São Francisco do Conde (BA), Brasil}

Maria Cristina da Silveira Galan Fernandes Universidade Federal de São Carlos - UFSCar, São Carlos (SP), Brasil

\section{Maria Helena Santos \\ Instituto Universitário de Lisboa - IUL, \\ Lisboa, Portugal}

Maria Ligia Coelho Prado

Universidade de São Paulo - USP,

São Paulo (SP), Brasil

Maria Regina Maluf

Pontifícia Universidade Católica de São Paulo - PUC-SP,

São Paulo (SP), Brasil 
Maria Rosa Lombardi

Fundação Carlos Chagas - FCC,

São Paulo (SP), Brasil

Maria Teresa Gonzaga Alves

Universidade Federal de Minas Gerais - UFMG,

Belo Horizonte (MG), Brasil

Marie Thériault

Université de Montréal - UdeM,

Québec, Canadá

Marília Bárbara Fernandes Garcia Moschko

Universidade Estadual de Campinas - Unicamp,

Campinas (SP), Brasil

Marília Pinto de Carvalho

Universidade de São Paulo - USP,

São Paulo (SP), Brasil

Maristela Rossato

Universidade de Brasilia - UnB,

Brasilia (DF), Brasil

Mary Ângela Teixeira Brandalise

Instituto Nacional de Estudos e Pesquisas Educacionais

Anisio Teixeira - Inep,

Brasilia (DF), Brasil

Menga Lüdke

Pontificia Universidade Católica do Rio de Janeiro - PUC-Rio,

Rio de Janeiro (RJ), Brasil

Michelle de Freitas Bissoli

Universidade Federal do Amazonas - UFAM,

Manaus (AM), Brasil

Miriam Fábia Alves

Universidade Federal de Goiás - UFG,

Goiânia (GO), Brasil

Mirian Jorge Warde

Pontifícia Universidade Católica de São Paulo - PUC-SP,

São Paulo (SP), Brasil

\section{Mitsuko Aparecida Makino Antunes}

Pontifícia Universidade Católica de São Paulo - PUC-SP,

São Paulo (SP), Brasil

Moema Guedes

Universidade Federal Rural do Rio de Janeiro - UFRRJ,

Seropédica (RJ), Brasil

Moema de Poli Teixeira

Fundação Cesgranrio,

Rio de Janeiro (RJ), Brasil
Murillo Marschner Alves de Brito

Pontifícia Universidade Católica do Rio de Janeiro - PUC-Rio,

Rio de Janeiro (RJ), Brasil

Nathalie Reis Itaborai

Universidade Federal de Juiz de Fora - UFJF,

Juiz de Fora (MG), Brasil

Neide Luzia de Rezende

Universidade de São Paulo - USP,

São Paulo (SP), Brasil

Neusa Maria Dal Ri

Universidade Estadual Paulista "Júlio de Mesquita Filho" - Unesp,

Marilia (SP), Brasil

Pablo Santaolalla Rueda

Universidad Miguel Hernández-UMH,

Alicante, España

Patrícia Cristina Albieri de Almeida

Fundação Carlos Chagas - FCC,

São Paulo (SP), Brasil

Paula Regina Costa Ribeiro

Universidade Federal do Rio Grande do Sul - UFRGS,

Porto Alegre (RS), Brasil

Paulo Pires de Queiroz

Universidade Federal Fluminense - UFF,

Niterói (RJ), Brasil

Raquel Souzas

Universidade Federal da Bahia - UFBA,

Vitória da Conquista (BA), Brasil

Raquel da Cunha Valle

Fundação Carlos Chagas - FCC,

São Paulo (SP), Brasil

Ricardo Gonçalves Severo

Universidade Federal do Rio Grande - FURG,

Rio Grande (RS), Brasil

Rosana Rodrigues Heringer

Universidade Federal do Rio de Janeiro - UFRJ,

Rio de Janeiro (RJ), Brasil

Samuel Mendonça

Pontifícia Universidade Católica de Campinas - PUC-Campinas,

Campinas (SP), Brasil

Sandra Zákia L. Sousa

Universidade de São Paulo - USP,

São Paulo (SP), Brasil 


\section{Sandra Palma Saleiro}

Instituto Universitário de Lisboa - ISCTE,

Lisboa, Portugal

\section{Sandra Mara Garcia}

Centro Brasileiro de Análise e Planejamento, Área de

População e Sociedade - Cebrap,

São Paulo (SP), Brasil

\section{Sílvia Maria Agatti Lüdorf}

Universidade Federal do Rio Grande do Norte-UFRN,

Natal (RN), Brasil

Simone Sehnem

Universidade do Oeste de Santa Catarina - Unoesc,

Joaçaba (SC), Brasil

\section{Sofia Viseu}

Universidade de Lisboa - ULisboa,

Lisboa, Portugal

Sonia Maria Chaves Haracemiv

Universidade Federal do Paraná-UFPR,

Curitiba (PR), Brasil

Sonia Teresinha de Sousa Penin

Universidade de São Paulo - USP,

São Paulo (SP), Brasil

Tatiana Arnaud Coutinho Cipiniuk

Universidade Federal Fluminense - UFF,

Niterói (RJ), Brasil

Teresa Leite

Instituto Politécnico de Lisboa - ISEL,

Lisboa, Portugal

Teresa Kleba Lisboa

Universidade Federal de Santa Catarina - UFSC,

Florianópolis (SC), Brasil

Thays Almeida Monticelli

Themis - Gênero, Justiça e Direitos Humanos,

Porto Alegre (RS), Brasil

Theresa Adrião

Universidade Estadual de Campinas - Unicamp,

Campinas (SP), Brasil

Thiago Magela Rodrigues Dias

Centro Federal de Educação Tecnológica de Minas Gerais -

Cefet- $M G$,

Belo Horizonte (MG), Brasil

Vanda Mendes Ribeiro

Universidade Cidade de São Paulo - Unicid,

São Paulo (SP), Brasil
Vanessa Ferraz Almeida Neves

Universidade Federal de Minas Gerais - UFMG,

Belo Horizonte (MG), Brasil

Vera Lucia Felicetti

Universidade La Salle - Unilasalle,

Canoas (RS), Brasil

Vera Maria Nigro de Souza Placco

Pontificia Universidade Católica de São Paulo - PUC-SP,

São Paulo (SP), Brasil

Wagner Rodrigues Silva

Universidade Federal do Tocantins (UFT),

Palmas (TO), Brasil

Wanda Maria Junqueira de Aguiar

Pontifícia Universidade Católica de São Paulo - PUC-SP,

São Paulo (SP), Brasil

Wânia Pasinato

Universidade de São Paulo - USP,

São Paulo (SP), Brasil

Wilma de Nazaré Baia Coelho

Universidade Federal do Pará - UFPA,

Belém (PA), Brasil

Wivian Weller

Universidade de Brasilia - UnB,

Brasilia (DF), Brasil

Zara Figueiredo Tripodi

Universidade Federal de Ouro Preto - UFOP,

Ouro Preto (MG), Brasil 